\title{
Sensibilidad a la ansiedad y adicción a los videojuegos en deportistas. El rol protector de la dureza mental.
}

\section{Anxiety sensitivity and video game addiction in athletes. The protective role of mental toughness.}

\section{Sensibilidade à ansiedade e dependência de jogos de vídeo em atletas. $O$ papel protector da resistência mental.}

\author{
López-Mora, C. ${ }^{1}$, Álvarez, O. ${ }^{2}$, González-Hernádez, J. ${ }^{3}$, Castillo, I. ${ }^{2}$ \\ ${ }^{1}$ Universidad Europea de Valencia, Valencia (España); ${ }^{2}$ Universitat de Valéncia, Valencia (España); \\ ${ }^{3}$ Universidad de Granada, Granada (España)
}

\section{RESUMEN}

El uso excesivo de los videojuegos y la adicción a los mismos entre los deportistas es una preocupación reciente que va en aumento. La sensibilidad a la ansiedad es un factor de riesgo para las conductas adictivas, del mismo modo que la dureza mental, ampliamente asociada a los deportistas, es un elemento protector. El presente trabajo examina las relaciones entre la sensibilidad a la ansiedad, la dureza mental y la adicción a videojuegos en este contexto. La muestra estuvo compuesta por deportistas $(n=98)$ de entre $19-51$ años $(\mathrm{M}$ edad $=23.94$; DT = 7.72) de los cuales 64 fueron hombres y 34 mujeres. Aplicando instrumentos descriptivos sobre sensibilidad a la ansiedad, dureza mental y adicción y dependencia a los videojuegos los resultados indicaron que un aumento de la sensibilidad a la ansiedad física o social correlacionó con una disminución de la dureza mental y la reducción de ésta se asoció con un aumento de la tolerancia a los video juegos. La sensibilidad a la ansiedad social y física tuvo efectos indirectos en el abuso y tolerancia a los videojuegos mediante la dureza mental de los deportistas. Los resultados se discuten entorno al papel de la dureza mental en la comprensión de los problemas de adicción a los videojuegos en deportistas.

Palabras clave: Adicción, Videojuegos, Deporte, Dureza Mental, Ansiedad.

\section{ABSTRACT}

Video game overuse and addiction among athletes is a recent and growing concern. Anxiety sensitivity is a risk factor for addictive behaviors, just as mental toughness, widely associated with athletes, is a protective element. The present study examines the relationships between anxiety sensitivity, mental toughness and video game addiction in this context. The sample was composed of athletes $(n=98)$ aged 19-51 years $(\mathrm{M}$ age $=23.94 ; \mathrm{SD}=7.72)$ of whom 64 were male and 34 females. Applying descriptive instruments on anxiety sensitivity, mental toughness and video game addiction and dependence, the results indicated that an increase in physical or social anxiety sensitivity correlated with a decrease in mental toughness that in turn was associated with an increase in video game tolerance. Sensitivity to social and physical anxiety had indirect effects on video game abuse and tolerance through mental toughness in athletes. The results are discussed around the role of mental toughness in understanding video game addiction problems in athletes.

Keywords: Addiction, Videogames, Sport, Mental Toughness, Anxiety. 


\section{Sensibilidad a la ansiedad dureza mental y adicción a videojuegos en deportistas}

\section{RESUMO}

O uso excessivo e a dependência dos jogos de vídeo entre os atletas é uma preocupação recente e crescente. A sensibilidade à ansiedade é um facto de risco para comportamentos viciantes, tal como a resistência mental, amplamente associada aos atletas, é um elemento protetor. O presente estudo examina as relações entre a sensibilidade à ansiedade, resistência mental e dependência de jogos de vídeo neste contexto. A amostra foi composta por atletas $(\mathrm{n}=98)$ com idades entre 19-51 anos $(\mathrm{M}$ idade =23,94; T.D. =7,72) dos quais 64 eram do sexo masculino, 34 do sexo feminino. Aplicando instrumentos descritivos sobre a sensibilidade à ansiedade, resistência mental, dependência e dependência de jogos de vídeo, os resultados indicaram que um aumento da sensibilidade à ansiedade física ou social correlacionada com uma diminuição da resistência mental, e esta diminuição foi associada a um aumento da tolerância aos jogos de vídeo. A sensibilidade à ansiedade social e física teve efeitos indiretos no abuso e tolerância dos jogos de vídeo através da resistência mental dos atletas. Os resultados são discutidos em torno do papel da resistência mental na compreensão dos problemas de vício dos jogos de vídeo nos atletas.

Palavras chave: Adição, Videogames, Desporto, Dureza Mental, Ansiedade.

\section{INTRODUCCIÓN}

En la última década, los videojuegos han aumentado su presencia en el día a día de los jóvenes (Kahn et al., 2015; Ward, 2018), siendo tendencia al alza debido al uso de dispositivos móviles para jugar (Orús, 2020) y la proliferación de juegos en red a través de internet (Dalisay et al., 2015; Vella et al., 2019). Abigail Orús (2020) señala que esta "nueva" forma de ocio ocupa de media 6.7 horas semanales entre los jugadores españoles llegando, en algunos casos, a convertirse en adicción (Gentile, 2009; Gentile et al., 2017). Se estima que entre un $5 \%$ y un $8 \%$ de los jugadores habituales desarrollan trastorno (Coyne et al., 2020; Gentile, 2009; Mentzoni et al., 2011), y entre un 3\% y un $13 \%$ si se trata de adultos jóvenes (Wartberg et al., 2020; Yang et al., 2020). Además, se ha detectado que los hombres se muestran más proclives al uso de videojuegos en línea y les dedican significativamente más tiempo a estas actividades frente a las mujeres, que prefieren no jugar o tienden a hacer un uso de internet más orientado hacia actividades relacionadas con la comunicación interpersonal, estando estas relaciones mediadas por la edad (Bonanno y Kommers, 2005; Weiser, 2000).

En los últimos tiempos el crecimiento de este recurso para el ocio y su amplia oferta en cuanto a contenido $\mathrm{y}$ formato ha llegado a todo tipo de poblaciones y segmentos, incluido el de los deportistas. De hecho, diferentes autores (Fortier, 2018; Sanderson et al., 2020; Sepkowitz, 2018) señalan a los deportistas como el principal grupo consumidor de videojuegos de tipo cooperativo en línea (e.g., Fortnite, juego cooperativo online diseñado como un juego de jugador sobre el entorno; Fortier, 2018), invirtiendo grandes cantidades de tiempo conectados con los miembros del propio equipo y sus seguidores (Sepkowitz, 2018). Todo ello, está generando preocupación entre la comunidad de técnicos sobre las consecuencias de estas prácticas e impacto en el rendimiento de los deportistas (Fortier, 2018).

El uso problemático de videojuegos (o adicción a videojuegos) se encuadra dentro de las adicciones conductuales, y se define como un patrón desordenado de comportamiento de juego persistente o recurrente ('juego digital' o 'videojuegos'), que puede darse en línea (i.e., Internet) o fuera de línea, al menos durante 12 meses (o menos cuando se cumplen todos los criterios de diagnóstico y los síntomas son graves; OMS, 2018). La dependencia generada no puede explicarse por ninguna actividad biológica o bioquímica, sino que es psicológica (motivacional, afectiva, condicionada, etc.) lo que indica que ciertos procesos psicológicos están relacionados con la manifestación de la dependencia (OMS, 2018).

Aunque todavía no hay consenso sobre la cantidad de síntomas necesarios para el diagnóstico de adicción (Gunuc, 2015) o sobre si todos los síntomas tienen la misma importancia (Blinka y Smahel, 2011), se observa que el uso problemático de videojuegos comparte algunos puntos comunes con respecto a la sintomatología, prevención y el tratamiento de otras adicciones conductuales (Griffiths, 2008a; Griffiths, 2008b; Griffiths, 2010; Gunuc, 2015; Keepers, 1990; Kuczmierczyk et al., 1987), como por ejemplo, alteraciones del estado de ánimo, preocupación 


\section{López-Mora et al.}

excesiva por el juego, presencia de mentiras o acciones de ocultación sobre el uso de videojuegos, perdida de interés en otras actividades, actitudes de defensa y enfado, retraimiento social y psicológico, tolerancia (aumento progresivo de la necesidad de jugar más o por más tiempo), abstinencia (irritabilidad, ansiedad o tristeza ante la retirada del videojuego sin origen farmacológico), uso de videojuegos como vía de escape de otras situaciones, uso excesivo, o consecuencias negativas en la vida diaria (DSM-V, 2013).

En este sentido, Choliz y Marco (2011) detallan una serie de indicadores de relevancia para la detección del uso problemático de videojuegos como: a) existencia de una necesidad por jugar más juegos o más tiempo que aumenta progresivamente, ya que los patrones de juego dejan de ser suficientes para el jugador/a, b) presencia de angustia emocional derivada de la interrupción o cese prolongado del juego, c) inversión de más tiempo en jugar del planificado en un inicio, d) incapacidad o dificultad para dejar de jugar, aun cuando existe la intención para dejar de hacerlo, e) las actividades de juego o relacionadas con el juego interfieren, interrumpen o dificultan actividades diarias y f) aun cuando el jugador es consciente de lo dañino o incorrecto de la situación, continua jugando.

Ser propenso a la ansiedad es un factor de riesgo para las conductas adictivas, incluidos los problemas con videojuegos (Blaszczynski y Nower, 2002; Bristow, et al., 2018; Hodgins et al., 2012). Desde las teorías de aprendizaje social (Bandura, 1971), se entiende que los jóvenes que experimentan altos niveles de ansiedad y entran en contacto con videojuegos, "aprenden" que estas conductas de juego alivian sus emociones negativas y displacenteras al evadirlos temporalmente de las preocupaciones y miedos que generan los estados de ansiedad. Estas conductas de evitación y escape de emociones y estados displacenteros a través de los videojuegos mantenidas en el tiempo, aumentan la probabilidad de transformar una actividad de ocio en una actividad adictiva y problemática (Blaszczynski y Nower, 2002; Bristow, et al., 2018; Thomas et al., 2009).

Por otro lado, desde los modelos psicobiológicos de adicción y ansiedad (Lüthi y Lüscher, 2014), también se apoya la hipótesis de que la conducta de juego, que en un principio genera recompensa (reducción de ansiedad), desencadena formas específicas de plasticidad sináptica, que en sujetos susceptibles se volvería persistente y conduciría a la enfermedad (Lüthi y Lüscher, 2014), lo que explica la alta comorbilidad entre los trastornos de ansiedad y adicción (Grant et al.,2006; Merikangas et al., 1998) y sugiere una superposición en los mecanismos neuronales subyacentes (Lüthi y Lüscher, 2014). En consonancia con estos planteamientos, una gran cantidad de literatura demuestra las asociaciones positivas entre la ansiedad y la adicción a videojuegos (e.g., Goel et al., 2013; Hyun et al., 2015; Van Rooij et al., 2011), así como las relaciones especificas existentes entre esta respuesta desadaptativa y la ansiedad estado/rasgo, donde la ansiedad rasgo presenta un gran tamaño del efecto sobre la adicción a videojuegos (Cole y Holley, 2013) convirtiendo así a las personas con problemas previos de ansiedad más susceptibles a presentar características disfuncionales del comportamiento del juego digital (Kessler et al., 2008; Männikkö et al., 2015).

Si bien la presencia de ansiedad ha demostrado ser un elemento a tener en cuenta en la comprensión de las conductas adictivas, en los últimos tiempos se está teniendo en cuenta la sensibilidad a la ansiedad como variable transdiagnóstica con capacidad para amplificar las respuestas de ansiedad (e.g., taquicardia, falta de aliento, rumiaciones, aislamiento social, etc.), que está motivada por el miedo que la persona presenta al anticipar la aparición de estos síntomas (Molina et al., 2014; Woodman y Hardy, 2001), y que explicaría por qué las personas con un alto grado de miedo a la ansiedad tienden a evitar ciertas actividades en las que anticipan el malestar, que les puede producir la aparición de la sintomatología ansiosa (Taylor et al., 2007). En este sentido también se ha demostrado que la anticipación de las sensaciones de ansiedad predice significativamente la presencia de conductas compatibles con la adicción. En general, la literatura demuestra que los altos niveles de ansiedad predisponen a las personas a tener problemas de juego, pero se ha hecho poco para comprender los mecanismos de esta asociación y mucho menos por entender los mecanismos de protección (Keough et al., 2015; Stewart y Kushner, 2001).

Otro elemento a considerar en la comprensión de las conductas adictivas sería la dureza mental (Bristow, et al., 2018). Según la aproximación de Clough y 


\section{Sensibilidad a la ansiedad dureza mental y adicción a videojuegos en deportistas}

colaboradores (2002) las personas con un alto componente de dureza mental son aquellas que: (1) perciben las barreras u obstáculos como pruebas en lugar de cargas, permitiéndoles adoptar estrategias eficaces para hacer frente a la situación, que (2) se comprometen y esfuerzan para lograr el éxito y/o la persistencia en la consecución de sus objetivos, que (3) se hacen cargo de su propia vida, realizan cambios conscientes, eligen objetivos y hacen planes, y tienen la capacidad de manejar y usar las emociones para lograr éxito, así como (4) creen en sus propias habilidades independientemente de validación externa (Zalewska et al., 2019).

En general, la literatura señala que la dureza mental se relaciona con menores experiencias de ansiedad (Haghighi, y Gerber, 2019; Godoy-Izquierdo y Godoy, 2002), niveles más bajos de estrés percibido (Gerber et al., 2013a ; Gerber et al., 2013b), menos síntomas depresivos (Gerber, et al., 2013a; Gerber et al., 2013b; Mutz et al., 2017), niveles más bajos de agotamiento (Gerber et al., 2013a; Gerber et al. ., 2015; Godoy-Izquierdo y Godoy, 2002 ), los cuales son factores de riesgo para el desarrollo de la adicción a videojuegos (Bristow, et al., 2018; Blaszczynski y Nower, 2002; Thomas et al., 2009).

En los contextos deportivos, la dureza mental se ha considerado como un elemento de protección del deportista que permite a estos hacer frente a desafíos que ponen a prueba los propios recursos (Álvarez et al., 2018) gracias a que modera el estrés, ayuda a recuperar el equilibrio después de un fracaso y promueve la movilización de recursos especialmente cuando hay riesgos y desafíos (Crust y Keegan, 2010).

Otros autores definen la dureza mental como "una capacidad personal para ofrecer un alto rendimiento de forma regular a pesar de los distintos grados de exigencias situacionales" (Gucciardi y Hanton, 2015, p. 442). Pero poco se ha descrito sobre si este aspecto ayuda a los deportistas fuera del entorno deportivo a hacer frente a las adversidades que se le presentan en el día a día, como puede ser el riesgo de adicción a videojuegos.

Por todo ello, atendiendo a la novedad del fenómeno y la preocupación de la comunidad por las consecuencias negativas de los videojuegos entre los deportistas (Fortier, 2018; Sepkowitz, 2018) cabe la necesidad de cuestionarse, si efectivamente, entre los deportistas se presenta la adicción a los videojuegos, y qué rasgos de los deportistas pueden estar explicando estas relaciones y sus consecuencias asociadas. De ahí que, bajo un diseño de tipo exploratorio-descriptivo, no experimental (Hernández et al., 2010), el objetivo del presente estudio sea examinar las relaciones existentes entre la sensibilidad a la ansiedad de los deportistas, su dureza mental y la adicción a videojuegos, así como explorar el papel que estos elementos (ansiedad y dureza mental) juegan en la presencia de sintomatología asociada al uso problemático de videojuegos.

Tomando como referencia la literatura expuesta anteriormente y para dar respuesta al objetivo propuesto, se pone a prueba un modelo en el que se hipotetiza que la relación entre la sensibilidad a la ansiedad y las variables de adicción y tolerancia estará mediada por la dureza mental (véase Figura 1).

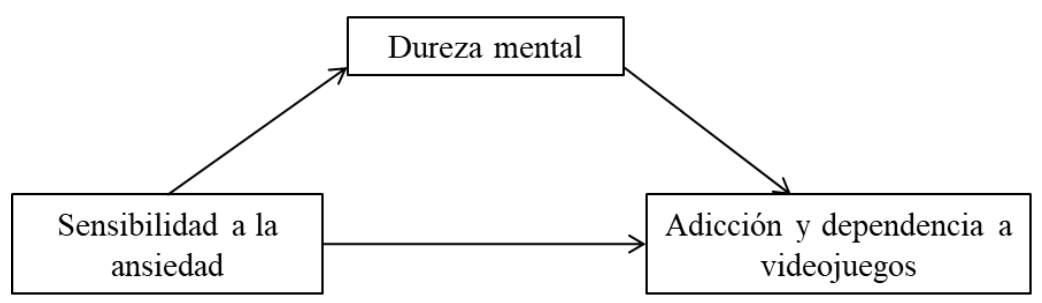

Figura 1. Modelo hipotetizado de la relación entre variables de sensibilidad a la ansiedad y variables de adicción y dependencia a videojuegos mediada por la dureza mental

\section{MATERIAL Y MÉTODOS}

\section{Participantes}

Los participantes fueron 98 deportistas (64 hombres, 34 mujeres; $M$ edad $=23.94$ años; D.T. $=7.72$, rango 19-51), practicantes de diferentes deportes (e.g., atletismo, fútbol, natación, etc.). La distribución por grupo de edad fue: 19-20 años $(\mathrm{n}=50), 21-25$ años (n $=24)$, y por encima de 26 años $(n=24)$. Por último, respecto al nivel o estatus económico, el $77.2 \%$ de los participantes informan tener unos ingresos por debajo de los 12.000 euros al año, un $6.5 \%$ se sitúa entre los 12.000 y los 20.000 euros al año y un $15.3 \%$ informaron tener unos ingresos por encima de los 20.000 euros al año.

\section{Instrumentos}

La sensibilidad a la ansiedad se evaluó con la versión española (Sandín et al., 2007) del cuestionario de 


\section{López-Mora et al.}

Índice de Sensibilidad a la Ansiedad-3 (ASI-3; Taylor et al., 2007). Este instrumento está compuesto por 18 ítems distribuidos en tres subescalas (con seis ítems cada una), que evalúan el factor cognitivo (e.g., "Cuando no puedo mantener mi mente concentrada en una tarea, siento la preocupación de que podría estar volviéndome loco/a"), físico (e.g., "Me asusto cuando mi corazón late de forma rápida") y social (e.g., "Para mí es importante no dar la impresión de estar nervioso/a") de la sensibilidad a la ansiedad. El deportista ha de contestar, indicando el grado en el que suele experimentar miedo/ansiedad ante cada uno de los síntomas de tipo cognitivo, físico y social, en una escala tipo Likert que oscila desde 1 (nada o casi nada) a 5 (muchísimo). Investigaciones previas han mostrado que el ASI-3 es una medida psicométricamente sólida y válida (e.g., Sandín et al., 2007; Taylor et al., 2007).

La dureza mental se evaluó con la versión española (Álvarez et al., 2018) del Índice de Dureza Mental (MTI; Gucciardi, et al., 2015). Este instrumento se compone de ocho ítems que representan ocho facetas de la dureza mental (e.g., "Creo en mi capacidad para alcanzar mis metas"). Los participantes respondieron a cada ítem en una escala tipo Likert que oscila desde 1 (falso el $100 \%$ de las veces) a 7 (verdadero el $100 \%$ de las veces). Este instrumento ha mostrado fiabilidad y validez en investigaciones previas (e.g., Álvarez et al., 2018; Gucciardi et al., 2015).

La adicción a los videojuegos se midió con el Test de Dependencia de Videojuegos (VDT; Chóliz y Marco, 2011). Este instrumento se compone de 25 ítems que evalúan la adicción y la dependencia a los videojuegos a través de cuatro subescalas: abstinencia (cinco ítems: "Si no me funciona un videojuego, busco otro rápidamente para poder jugar"), abuso y tolerancia (cinco ítems: "juego mucho más tiempo con los videojuegos que cuando comencé"), problemas asociados con los videojuegos (cuatro ítems: "Me he acostado más tarde o he dormido menos por quedarme jugando con videojuegos") y dificultad en el control (seis ítems: "En cuanto tengo un poco de tiempo me pongo un videojuego, aunque sólo sea un momento"). A los participantes se les pregunta en qué medida están de acuerdo con las afirmaciones sobre el uso que hacen de los videojuegos, y las respuestas se recogen en una escala tipo Likert que oscila desde 1 (no, en absoluto) a 5 (si, totalmente de acuerdo). Este instrumento ha mostrado su fiabilidad y validez en investigaciones previas (e.g., Chóliz y Marco, 2011; Jiménez-Murcia et al., 2014).

\section{Procedimiento}

Los participantes fueron seleccionados por un muestreo de conveniencia tomando como criterio de inclusión el que participaran en alguna actividad deportiva y que fueran mayores de edad. Los deportistas contestaron a los cuestionarios, elaborados con el software Google Forms, a través de un link que se les proporcionó a través de las redes sociales. La participación fue voluntaria, se les pidió el consentimiento, y se les aseguró el anonimato y confidencialidad siguiendo los preceptos de la Declaración de Helsinki (2017). El tiempo invertido en la cumplimentación no excedió de los 10 minutos.

\section{Análisis estadístico}

Con el programa estadístico SPSS v21 (IBM, Co) se examinaron los datos faltantes (por debajo del $0.5 \%$ ) y no se hallaron valores atípicos univariados. Posteriormente se realizaron análisis descriptivos, diferenciales por sexo y edad, así como análisis de consistencia interna (alfa de Cronbach) de los instrumentos utilizados. Se utilizó la macro del PROCESS de Hayes y Scharkow (2013) para poner a prueba distintos modelos de mediación (modelo 4) para determinar si la relación entre la sensibilidad a la ansiedad y las variables de adicción y tolerancia estaba mediada por la dureza mental (véase Figura 1). Los errores estándar de los efectos indirectos se estimaron con el método de bootstrapping basado en 1000 muestras, considerándose significativo cuando el 95\% del intervalo de confianza no contiene el cero.

\section{RESULTADOS}

En la Tabla 1 se muestran las medias, desviaciones típicas, alfas de Cronbach y correlaciones entre todas las variables del estudio, así como con la edad, el sexo, el nivel educativo y económico de los participantes. Considerando la media escalar, los deportistas del estudio informaron de baja sensibilidad a la ansiedad, una alta dureza mental, y una baja adicción a los videojuegos. La consistencia interna de los instrumentos del estudio fue aceptable, oscilando los alfas de Cronbach entre .79 y .88 (véase Tabla 1). 


\section{Sensibilidad a la ansiedad dureza mental y adicción a videojuegos en deportistas}

Tabla 1. Estadísticos descriptivos, consistencia interna y correlaciones Pearson de las variables del estudio $(\mathrm{n}=98)$

\begin{tabular}{|c|c|c|c|c|c|c|c|c|c|c|c|}
\hline & Rango & M & $D T$ & 1 & 2 & 3 & 4 & 5 & 6 & 7 & 8 \\
\hline 1. Sensibilidad ansiedad cognitiva & $1-5$ & 2.21 & 0.75 & $(.83)$ & & & & & & & \\
\hline 2. Sensibilidad ansiedad física & $1-5$ & 2.51 & 0.88 & $.57 * *$ & $(.85)$ & & & & & & \\
\hline 3. Sensibilidad ansiedad social & $1-5$ & 2.85 & 0.81 & $.60 * *$ & $.52 * *$ & $(.79)$ & & & & & \\
\hline 4. Dureza mental & $1-7$ & 5.49 & 1.01 & -.22 & $-.34 *$ & $-.33 *$ & $(.86)$ & & & & \\
\hline 5. Abstinencia & $1-4$ & 1.64 & 0.66 & -.03 & .07 & .13 & -.16 & $(.88)$ & & & \\
\hline 6. Problemas uso excesivo & $1-4$ & 1.88 & 0.86 & -.04 & .06 & .08 & -.23 & $.76^{* *}$ & $(.84)$ & & \\
\hline 7. Dificultad en el control & $1-4$ & 1.69 & 0.67 & -.11 & .04 & -.02 & -.24 & $.85^{* *}$ & $.68 * *$ & $(.84)$ & \\
\hline 8. Abuso y tolerancia & $1-4$ & 1.48 & 0.67 & -.02 & .06 & .03 & $-.31 *$ & $.77 * *$ & $.54 * *$ & $.82 * *$ & $(.82)$ \\
\hline 9. Edad & - & 23.9 & 7.72 & .06 & .07 & -.09 & .04 & $-.35 * *$ & $-.46 * *$ & $-.30 * *$ & $-.28 * *$ \\
\hline 10. Sexo $(1=$ hombre; $2=$ mujer $)$ & - & - & - & -.06 & -.07 & -.02 & $.35^{*}$ & $-.33 * *$ & $-.31 * *$ & $-.37 * *$ & $-.25^{*}$ \\
\hline 11. Nivel económico & - & - & - & -.08 & -.02 & .03 & $.32 *$ & $-.29 * *$ & $-.28 * *$ & $-.33 * *$ & $-.21 *$ \\
\hline
\end{tabular}

Nota. Los alfas de Cronbach se muestran en la diagonal entre paréntesis. $* p<.05, * * p<.01$

La relación negativa y significativa entre la dureza mental y la sensibilidad a la ansiedad física y social fue moderada, sugiriendo que cuanta más dureza mental tienen los deportistas en menor medida informan de sensibilidad física y social. Asimismo, la dureza mental se relacionó negativa y significativamente con el abuso y tolerancia a los videojuegos, lo que sugiere que cuanto mayor es la dureza mental de los deportistas en menor medida refieren malestar por no poder usar los videojuegos. Por último, en cuanto a las variables sociodemográficas evaluadas, la edad, el sexo y el nivel económico se relacionaron moderadamente y de forma negativa y significativa con las variables de adicción y dependencia a los videojuegos. En concreto, el pertenecer a un grupo de menor edad, el ser hombre y tener un nivel de ingresos bajo se asoció a mayor adicción y dependencia a los videojuegos.

La prueba $t$ realizada para comparar a los hombres y mujeres mostraron que no existieron diferencias por sexo en las variables de sensibilidad a la ansiedad ni en la dureza mental (véase Tabla 2). Sin embargo, confirmando lo obtenido en las correlaciones, sí se observaron diferencias por sexo en las variables de adicción y dependencia a los videojuegos, siendo los hombres los que informaron en mayor medida que las mujeres sentir malestar cuando no pueden utilizar los videojuegos (abstinencia), tener más problemas ocasionados por los videojuegos (problemas uso excesivo), mayor dificultad en el control y mayor abuso y tolerancia a los videojuegos.

En la Tabla 3 se muestran los resultados del ANOVA realizado para examinar las posibles diferencias por grupos de edad, encontrándose diferencias significativas por edad en las variables de dureza mental y de adicción y dependencia a los videojuegos. Los resultados post hoc (Tukey HSD) mostraron que los mayores de 26 años respecto a los menores (19-20 años) y medianos (21-25 años) poseen mayor dureza mental $(\mathrm{M}=6.16 ; \mathrm{M}=5.34 ; \mathrm{M}=5.21$ respectivamente), así como menor abstinencia ( $\mathrm{M}=$ $1.28 ; \mathrm{M}=1.73 ; \mathrm{M}=1.81$ respectivamente), menores problemas por el uso excesivo de videojuegos $(\mathrm{M}=$ $1.43 ; \mathrm{M}=1.95 ; \mathrm{M}=2.18$ respectivamente) y menor dificultad en el control $(\mathrm{M}=1.28 ; \mathrm{M}=1.77 ; \mathrm{M}=1.92$ respectivamente). Por último, los deportistas entre 21 y 25 años en comparación con los más mayores (más de 26 años) informaron de mayor abuso y tolerancia a los videojuegos $(\mathrm{M}=1.72 ; \mathrm{M}=1.20$ respectivamente 


\section{López-Mora et al.}

Tabla 2. Diferencias por sexo en las variables del estudio

\begin{tabular}{lcccccc}
\hline & \multicolumn{5}{c}{$\begin{array}{c}\text { Hombres } \\
(\mathrm{n}=64)\end{array}$} & \multicolumn{3}{c}{$\begin{array}{c}\text { Mujeres } \\
(\mathrm{n}=34)\end{array}$} \\
\hline Sensibilidad ansiedad cognitiva & Rango & Media & $D T$ & Media & $D T$ & $t$ \\
Sensibilidad ansiedad física & $1-5$ & 2.18 & 0.72 & 2.27 & 0.81 & -0.59 \\
Sensibilidad ansiedad social & $1-5$ & 2.46 & 0.91 & 2.59 & 0.84 & -0.72 \\
Dureza mental & $1-5$ & 2.90 & 0.84 & 2.75 & 0.77 & 0.84 \\
Abstinencia & $1-7$ & 5.46 & 1.01 & 5.54 & 1.04 & -0.27 \\
Problemas uso excesivo & $1-4$ & 1.81 & 0.66 & 1.33 & 0.52 & $3.64^{* *}$ \\
Dificultad en el control & $1-4$ & 2.17 & 0.86 & 1.35 & 0.55 & $5.75^{* *}$ \\
Abuso y tolerancia & $1-4$ & 1.83 & 0.66 & 1.42 & 0.61 & $3.02^{* *}$ \\
\hline
\end{tabular}

Nota: $* * p<.01$.

Tabla 3. Diferencias por edad en las variables del estudio

\begin{tabular}{lcccccccc}
\hline & \multicolumn{3}{c}{$19-20$ años $(\mathrm{n}=50)$} & \multicolumn{2}{c}{$21-25$ años $(\mathrm{n}=24)$} & \multicolumn{2}{c}{-26 años $(\mathrm{n}=24)$} \\
\hline Sensibilidad ansiedad cognitiva & Rango & Media & $D T$ & Media & $D T$ & Media & $D T$ & $F$ \\
Sensibilidad ansiedad física & $1-5$ & 2.32 & .70 & 2.07 & .78 & 2.14 & .84 & 1.02 \\
Sensibilidad ansiedad social & $1-5$ & 2.62 & .82 & 2.42 & .89 & 2.35 & .98 & 0.91 \\
Dureza mental & $1-5$ & 2.99 & .81 & 2.66 & .86 & 2.76 & .77 & 1.53 \\
Abstinencia & $1-7$ & 5.35 & 1.06 & 5.21 & .83 & 6.16 & .88 & $3.19^{*}$ \\
Problemas uso excesivo & $1-4$ & 1.73 & .67 & 1.81 & .71 & 1.28 & .40 & $5.42^{* *}$ \\
Dificultad en el control & $1-4$ & 1.96 & .88 & 2.18 & .89 & 1.44 & .59 & $5.24 * *$ \\
Abuso y tolerancia & $1-4$ & 1.77 & .64 & 1.92 & .74 & 1.28 & .47 & $7.06^{* *}$ \\
\hline
\end{tabular}

Nota: $* p<.05, * * p<.01$.

Tabla 4. Resultados de los efectos de mediación de la dureza mental entre las variables de sensibilidad a la ansiedad y las variables de abuso y tolerancia a los videojuegos

\begin{tabular}{lcccc}
\hline & Dureza mental & Abuso y tolerancia & \\
Modelos & ED & ED & EI & $\mathrm{R}^{2}$ modelo total \\
\hline Sensibilidad ansiedad cognitiva & -.29 & -.02 & .06 & .06 \\
Sensibilidad ansiedad física & $-.41^{*}$ & .11 & $.06^{\mathrm{a}}$ & $.11^{*}$ \\
Sensibilidad ansiedad social & $-.45^{*}$ & .00 & $.08^{\mathrm{a}}$ & $.11^{*}$ \\
Mediador Dureza mental & & $-.19^{*}$ & & \\
\hline Nota. $S=$ sensibilidad; ED $=$ efecto directo EI = efecto indirecto. ${ }^{*} p<.05, a=$ efecto indirecto significativo \\
(intervalo de confianza no incluye el cero).
\end{tabular}

En la Tabla 4 se presentan los resultados de los análisis de regresión en los que se analiza si la dureza mental media la relación entre las variables de sensibilidad a la ansiedad y el abuso y la tolerancia a los videojuegos. La posible predicción en el resto de las variables de adicción y dependencia a los videojuegos (i.e., abstinencia, problemas uso excesivo y dificultad en el control) no se ha analizado, dado que, en las correlaciones previas, la asociación entre la dureza mental y dichas variables criterio no ha resultado estadísticamente significativa. La sensibilidad a la ansiedad física y social predice negativamente la dureza mental, la cual a su vez predice negativamente el abuso y la tolerancia a los videojuegos. Dichas sensibilidades (física y social) no predicen directamente el abuso y la tolerancia a los videojuegos, pero sí que la predicción es significativa a través de su relación con la dureza mental (véase Figura 2).

\section{DISCUSIÓN}

El propósito de este estudio fue examinar las asociaciones entre la adicción y dependencia a los videojuegos y la sensibilidad a la ansiedad, a través de las funciones mediadoras de la dureza mental en deportistas. Los resultados informan de los mecanismos intrapersonales asociados con el abuso y tolerancia a los videojuegos. 


\section{Sensibilidad a la ansiedad dureza mental y adicción a videojuegos en deportistas}
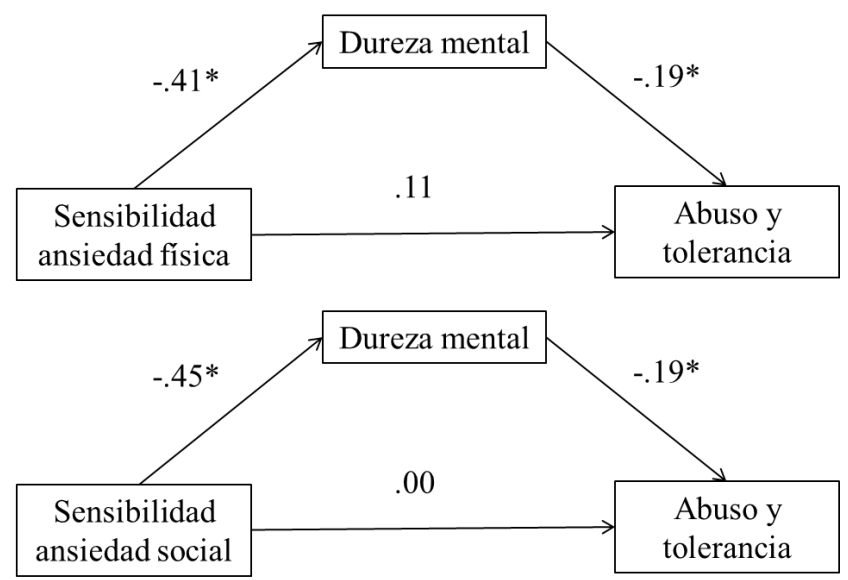

Figura 2. Solución no estandarizada del modelo de mediación. * $\mathrm{p}<.05$.

La sensibilidad a la ansiedad social estuvo indirectamente relacionada con el abuso y tolerancia a videojuegos a través de la afectación de la dureza mental. En otras palabras, los deportistas con miedo a que su ansiedad se perciba en un contexto social (e.g., ser percibido nervioso por otras personas) informaron de mayores síntomas de tolerancia y abuso de videojuegos, a través de una afectación de la percepción que tienen acerca de sus capacidades para hacer frente a situaciones de una forma eficaz. Por su parte, la sensibilidad a la ansiedad física también estuvo indirectamente relacionada con la adicción y dependencia a través del mismo mecanismo, siendo los deportistas con miedo a desarrollar síntomas ansiosos (e.g., aceleración del pulso, taquicardia, sudoración, etc.) quienes presentaron una mayor tolerancia y abuso mediado por una afectación de su dureza mental. Estas conclusiones son coherentes con la noción de, ante la aparición del miedo (e.g., miedo a la ansiedad), considerar que la personas con una baja dureza mental tenderán a activar conductas de escape y/o evasión para alejarse de aquellos estímulos ambientales o situaciones que están activando la experiencia de miedo (Adil et al., 2019). Mientras que los individuos mentalmente fuertes, ante el mismo estimulo, mantienen sus pensamientos positivos, practican el autocontrol constructivo, y evitan hábitos negativos (Adil et al., 2019).

Por otro lado, se corroboraron las relaciones entre la sensibilidad a la ansiedad y la dureza mental. Concretamente se pudo observar que existió una relación negativa entre los componentes de sensibilidad a la ansiedad física y social con la dureza mental de los deportistas. Estos resultados sugieren que deportistas comprometidos y esforzados para lograr sus objetivos, capaces de regular sus estados emocionales y conscientes y confiadas de sus propias habilidades, experimentan un menor miedo a la posibilidad de experimentar sensaciones físicas desagradables (e.g., falta de aire, aceleración del pulso, sudoración, etc.) y/o que estas sean percibidas por las personas de su entorno.

Estos resultados están en la línea de la literatura que señala relaciones negativas entre dureza mental y las experiencias de ansiedad en deportistas, demostrando que los deportistas que puntúan alto en las características de dureza mental también tienden a reportar bajos niveles de ansiedad en la competición (Gucciardi \& Jones, 2012; Haghighi, \& Gerber, 2019; Kristjánsdóttir, et al., 2018; Schaefer et al., 2016; Thelwell et al., 2005), así como aquella que relaciona la dureza mental con niveles más bajos de estrés percibido (Gerber et al., 2013a; Gerber et al., 2013b). La asociación positiva encontrada entre la dureza mental y la sensibilidad a la ansiedad es coherente con las definiciones conceptuales de la dureza mental, que afirman que una característica esencial de los deportistas mentalmente fuertes es su capacidad para manejar las emociones desagradables, como la ansiedad de la competición (Thelwell et al., 2005).

Además, tal y como se esperaba, se confirmaron las relaciones entre la dureza mental y la adicción a videojuegos, concretamente con el elemento de abuso $\mathrm{y}$ tolerancia, encontrando asociaciones negativas entre ambos elementos, lo que sugiere que cuanto mayor es la dureza mental de los deportistas en menor medida refieren malestar por no poder usar los videojuegos y no experimentan un aumento progresivo de la necesidad de jugar más o por más tiempo. Si bien no existe literatura previa que muestre las relaciones especificas entre la dureza mental y la adicción a videojuegos en deportistas, Soleimani, y colaboradores (2016), mostraron un patrón consistente en el que estudiantes que tenían una alta tendencia a la adicción presentaban bajos niveles en dureza y apoyan sus conclusiones en el hecho de que la dureza mental es un escudo que proporciona un mecanismo de defensa para el comportamiento de afrontamiento cuando una persona se enfrenta a acontecimientos vitales negativos o a condiciones de vida adversas.

En la misma línea, Wang et al. (2014), examinaron la relación entre la adicción a Internet y la dureza mental 


\section{López-Mora et al.}

en estudiantes universitarios encontrando relaciones en la línea de los resultados presentados en el presente trabajo, mostrando la dureza mental una correlación negativa con la adicción a Internet.

En cuanto a las variables sociodemográficas evaluadas, la edad, el sexo y el nivel económico se relacionaron moderadamente y de forma negativa y significativa con las variables de adicción $\mathrm{y}$ dependencia a los videojuegos. En concreto, los hombres menores de 26 años con un nivel de ingresos bajo se asociaron con mayores indicadores de abstinencia, problemas de uso excesivo, dificultades de control y mayores síntomas de abuso y tolerancia $\mathrm{o}$, dicho de otro modo, mayor adicción y dependencia a los videojuegos. Además, los varones tienden a presentar mayor riesgo de adicción a los videojuegos que las mujeres, en parte debido a la amplia diferencia de tiempo promedio que los chicos dedican a los videojuegos respecto a las chicas estando estas relaciones mediadas por la edad (Bonanno y Kommers, 2005; Hauge y Gentile, 2003; Weiser, 2000; Wang, et al., 2014) siendo la tasa de adicción a Internet de los estudiantes universitarios de primer año más alta respecto a la tendencia de adicción de los estudiantes de último año (Wang, et al., 2014).

Estos hallazgos están en consonancia con literatura previa donde se señala que las etapas de adolescencia y adultez temprana son claves para la comprensión de la aparición de los trastornos adictivos (Kuss y Griffiths, 2012a,b). Diferentes autores señalan que estas etapas del desarrollo son especialmente críticas para la transformación patológica de la conducta de jugar a los videojuegos debido a la flexibilidad y autonomía para decidir dónde emplear su tiempo libre. Autonomía que los jóvenes adquieren al separarse progresivamente de las figuras de referencia paternas (y encaminarse hacia una vida adulta independiente y autónoma) sin tener aún construido un esquema sólido de personalidad y herramientas de afrontamiento para los contextos de riesgo (Stockdale y Coyne, 2018; Prot y Gentile 2014).

Estos resultados deben interpretarse a la luz de las limitaciones del estudio. En primer lugar, la medición de las variables mediante cuestionarios de autoinforme puede crear una varianza de método compartido y está sujeta a exigencias de auto-presentación. Por ello, es necesario realizar investigaciones futuras que utilicen métodos múltiples (por ejemplo, múltiples informantes, tareas de comportamiento) para reducir dichas fuentes. En segundo lugar, la presente muestra no es representativa de la población deportista en general. Es necesario investigar este tema con una muestra basada en la comunidad para determinar mejor la generalización de los resultados. Y, en tercer lugar, el diseño del estudio fue transversal, lo que no permite hacer inferencias sólidas sobre la causalidad y la dirección de los efectos y la importancia de otras variables relevantes no contempladas en el presente estudio como el apoyo familiar, el historial de adicción familiar, etc. Es posible, por ejemplo, ser deportista desde la niñez pudiera influir en el desarrollo de la dureza mental y habilidades para la gestión de la ansiedad. Por lo tanto, se necesitan diseños de estudio más rigurosos (por ejemplo, experimental, longitudinal prospectivo) que permitan inferencias de causalidad más fuertes.

A pesar de estas limitaciones del estudio, los presentes hallazgos amplían nuestra comprensión de las funciones de la sensibilidad a la ansiedad, la dureza mental y sus relaciones con las conductas de adicción a videojuegos que tanto preocupan en la actualidad. Dada la escasez de investigaciones que examinan estas relaciones, los resultados proporcionan pruebas de que la dureza mental podrían proteger contra los comportamientos de adicción a los videojuegos en los deportistas. Este trabajo informa que los deportistas que experimentan sensibilidad a la ansiedad social y que muestran niveles relativamente altos de dureza mental pueden evitar la necesidad de jugar a videojuegos más y por más tiempo. Los académicos han señalado que minimizar los riesgos asociados para los jóvenes de la generación digital se ha convertido en un objetivo importante de los responsables políticos en los últimos años (Rideout y Robb, 2019; Vondráčková y Gabrhelík, 2016). Aunque se necesita más investigación, los desarrolladores de programas preventivos y los responsables políticos deberían considerar el apoyo a las intervenciones que fomentan la práctica deportiva regular para abordar este objetivo.

\section{APLICACIONES PRÁCTICAS}

Los presentes hallazgos resultan de interés y extrema utilidad para aquellos profesionales de la psicología que trabajan en el ámbito del deporte cara a la planificación de intervenciones específicas sobre la prevención de conductas adictivas, ya que se trata de 


\section{Sensibilidad a la ansiedad dureza mental y adicción a videojuegos en deportistas}

hacer uso de variables que puedan ayudar a la prevención. En este sentido, se recomienda incluir trabajos específicos en fortaleza mental, ya que se muestra como un protector relevante y significativo entre variables disposicionales (sensibilidad a la ansiedad física y social) y la adicción y dependencia a los videojuegos que tanto preocupa entre la comunidad de técnicos sobre las consecuencias de estas prácticas e impacto en el rendimiento de los deportistas.

\section{REFERENCIAS}

1. Adil, A., Jabeen, N., \& Yousaf, A. (2019). Mediating role of mental toughness between behavioral systems and depression. Pakistan Journal of Medical Research, 58(2), 51-58.

2. Álvarez, O., Walker, B., \& Castillo, I. (2018). Examining motivational correlates of mental toughness in Spanish athletes. Cuadernos de Psicología del Deporte, 18(1), 141-150.

3. Bandura, A. (1971). Social learning theory. General Learning Press.

4. Blaszczynski, A., \& Nower, L. (2002). A pathways model of problem and pathological gambling. Addiction, 97(5), 487-499. https://doi.org/10.1046/j.1360-

0443.2002.00015.x

5. Blinka, L., \& Šmahel, D. (2011). Predictors of adolescents' excessive internet use: A comparison across European countries. 15th European Conference on Developmental Psychology (337-341). Medimond.

6. Bonanno, P., \& Kommers, P. A. (2005). Gender differences and styles in the use of digital games. Educational Psychology, 25(1), 13-41.

https://doi.org/10.1080/01443410420002948 77

7. Bristow, L. A., Bilevicius, E., Stewart, S. H., Goldstein, A. L., \& Keough, M. T. (2018). Solitary gambling mediates the risk pathway from anxiety sensitivity to excessive gambling: Evidence from a longitudinal ecological momentary assessment study. Psychology of Addictive Behaviors, 32(6), 689-696. https://doi.org/10.1037/adb0000395

8. Chóliz, M., y Marco, C. (2011). Patrón de uso $\mathrm{y}$ dependencia de videojuegos en infancia y adolescencia. Anales de Psicología, 27(2), 418-426.

9. Clough, P., Earle, K., \& Sewell, D. (2002). Mental toughness: The concept and its measurement. Solutions in Sport Psychology, 32-43.

10. Cole, S. H., \& Hooley, J. M. (2013). Clinical and personality correlates of MMO gaming: Anxiety and absorption in problematic internet use. Social science computer review, $31(4)$, 424-436. https://doi.org/10.1177/0894439312475280

11. Coyne, S. M., Stockdale, L. A., Warburton, W., Gentile, D. A., Yang, C., \& Merrill, B. M. (2020). Pathological video game symptoms from adolescence to emerging adulthood: A 6year longitudinal study of trajectories, predictors, and outcomes. Developmental Psychology, 56(7), 1385-1396. https://doi.org/10.1037/dev0000939

12. Crust, L., \& Keegan, R. (2010). Mental toughness and attitudes to risk-taking. Personality and Individual Differences, 49(3), 164-168.

https://doi.org/10.1016/j.paid.2010.03.026

13. Dalisay, F., Kushin, M. J., Yamamoto, M., Liu, Y. I., \& Skalski, P. (2015). Motivations for game play and the social capital and civic potential of video games. New Media \& Society, 17(9), 1399-1417. https://doi.org/10.1177/1461444814525753

14. Fortier, A. (2018). Making meaning out of social harm in videogames (Doctoral dissertation). Arts \& Social Sciences: School of Criminology.

15. Fortier, S. (20 de Julio de 2018) Are pro athletes playing too much Fortnite? Some teams are worried. The Washington Post. https://www.washingtonpost.com/sports/arepro-athletes-playing-too-much-fortnite-someteams-are-worried/2018/07/20/664d5ffe8ab5-11e8-85ae-511bc1146b0b_story.html

16. Gentile, D. (2009). Pathological video-game use among youth ages 8 to 18: A national study. Psychological science, 20(5), 594-602. https://doi.org/10.1111/j.14679280.2009.02340.x 


\section{López-Mora et al.}

17. Gentile, D. A., Bailey, K., Bavelier, D., Brockmyer, J. F., Cash, H., Coyne, S. M., ... \& Young, K. (2017). Internet gaming disorder in children and adolescents. Pediatrics, 140(Supplement 2), S81-S85. https://doi.org/10.1542/peds.2016-1758h

18. Gerber, M., Brand, S., Feldmeth, A. K., Lang, C., Elliot, C., Holsboer-Trachsler, E., \& Pühse, U. (2013a). Adolescents with high mental toughness adapt better to perceived stress: A longitudinal study with Swiss vocational students. Personality and Individual Differences, 54(7), 808-814.

19. Gerber, M., Kalak, N., Lemola, S., Clough, P. J., Perry, J. L., Pühse, U., ... \& Brand, S. (2013b). Are adolescents with high mental toughness levels more resilient against stress?. Stress and Health, 29(2), 164-171. https://doi.org/10.1016/j.paid.2012.12.003

20. Goel, D., Subramanyam, A., \& Kamath, R. (2013). A study on the prevalence of internet addiction and its association with psychopathology in Indian adolescents. Indian Journal of Psychiatry, 55(2), 140. https://doi.org/10.4103/0019-5545.111451

21. Grant, B. F., Stinson, F. S., Dawson, D. A., Chou, S. P., Dufour, M. C., Compton, W., ... \& Kaplan, K. (2006). Prevalence and cooccurrence of substance use disorders and independent mood and anxiety disorders: Results from the National Epidemiologic Survey on Alcohol and Related Conditions. Alcohol Research \& Health, 29(2), 107. https://doi.org/10.1001/archpsyc.61.8.807

22. Griffiths, M. D. (2008a). Diagnosis and management of video game addiction. New directions in addiction treatment and prevention (12), 27-41.

23. Griffiths, M. D. (2008b). Internet and videogame addiction. En C. Essau (Ed.), Adolescent addiction: Epidemiology, assessment and treatment (pp. 231-267). Elsevier.

24. Griffiths, M. D. (2010). The role of context in online gaming excess and addiction: Some case study evidence. International Journal of Mental Health and Addiction, 8(1), 119-125. https://doi.org/10.1007/s11469-009-9229-x

25. Godoy-Izquierdo, D., \& Godoy, J. F. (2002). La personalidad resistente: una revisión de la conceptualización e investigación sobre la dureza. Clínica y salud, 13(2), 135-162.

26. Gucciardi, D. F., \& Jones, M. I. (2012). Beyond optimal performance: Mental toughness profiles and developmental success in adolescent cricketers. Journal of Sport and exercise Psychology, 34(1), 16-36. https://doi.org/10.1123/jsep.34.1.16

27. Gucciardi, D. F., Hanton, S., Gordon, S., Mallett, C. J., \& Temby, P. (2015). The concept of mental toughness: Tests of dimensionality, nomological network, and traitness. Journal of Personality, 83(1), 26-44. https://doi.org/10.1111/jopy.12079

28. Gunuc, S. (2015). Relationships and associations between video game and Internet addictions: Is tolerance a symptom seen in all conditions. Computers in Human Behavior, 49 , 517-525.

https://doi.org/10.1016/j.chb.2015.03.063

29. Haghighi, M., \& Gerber, M. (2019). Does mental toughness buffer the relationship between perceived stress, depression, burnout, anxiety, and sleep? International Journal of Stress Management, 26(3), 297305. https://doi.org/10.1037/str0000106

30. Hauge, M. R., \& Gentile, D. A. (2003, April). Video game addiction among adolescents: Associations with academic performance and aggression. In Society for Research in Child Development Conference.

31. Hayes, A. F., \& Scharkow, M. (2013). The relative trustworthiness of inferential tests of the indirect effect in statistical mediation analysis: Does method really matter? Psychological Science, 24(10), 1918-1927. https://doi.org/10.1177/0956797613480187

32. Hernández, R., Fernández, C., \& Baptista, P. (2010). Metodología de la investigación ( $5^{\mathrm{a}}$ ed.). McGraw-Hill Interamericana.

33. Hodgins, D. C., Schopflocher, D. P., Martin, C. R., el-Guebaly, N., Casey, D. M., Currie, S. R., ... \& Williams, R. J. (2012). Disordered gambling among higher-frequency gamblers: who is at risk? Psychological medicine, $42(11)$, 2433. https://doi.org/10.1017/s0033291712000724

34. Hyun, G. J., Han, D. H., Lee, Y. S., Kang, K. D., Yoo, S. K., Chung, U. S., \& Renshaw, P. 


\section{Sensibilidad a la ansiedad dureza mental y adicción a videojuegos en deportistas}

F. (2015). Risk factors associated with online game addiction: A hierarchical model. Computers in human behavior, 48, 706-713. https://doi.org/10.1016/j.chb.2015.02.008

35. Jiménez-Murcia, S., Fernández-Aranda, F., Granero, R., Chóliz, M., La Verde, M., Aguglia, E.,... \& del Pino-Gutiérrez, A. (2014). Video game addiction in gambling disorder: Clinical, psychopathological, and personality correlates. BioMed Research International (14)1, 11, https://doi.org/10.1155/2014/315062

36. Kahn, A. S., Shen, C., Lu, L., Ratan, R. A., Coary, S., Hou, J., ... \& Williams, D. (2015). The Trojan Player Typology: A cross-genre, cross-cultural, behaviorally validated scale of video game play motivations. Computers in Human Behavior, 49, 354-361. https://doi.org/10.1016/j.chb.2015.03.018

37. Keepers, G. A. (1990). Pathological preoccupation with video games. Journal of the American academy of child \& adolescent psychiatry, 29(1), 49-50. https://doi.org/10.1097/00004583199001000-00009

38. Keough, M. T., Hines, S., Winslade, A., \& O'Connor, R. M. (2015). Negative urgency and gender moderate the association between anxiety sensitivity and alcohol-related problems. Journal of Addiction \& Prevention, $3(1), \quad 1-7 . \quad$ https://doi.org/10.13188/23302178.1000018

39. Kessler, R. C., Hwang, I., LaBrie, R., Petukhova, M., Sampson, N. A., Winters, K. C., \& Shaffer, H. J. (2008). The prevalence and correlates of DSM-IV pathological gambling in the National Comorbidity Survey Replication. Psychological medicine, 38(9), 1351.

https://doi.org/10.1017/s0033291708002900

40. Kristjánsdóttir, H., Erlingsdóttir, A. V., Sveinsson, G., \& Saavedra, J. M. (2018). Psychological skills, mental toughness and anxiety in elite handball players. Personality and Individual Differences, 134, 125-130. https://doi.org/10.1016/j.paid.2018.06.011

41. Kuczmierczyk, A. R., Walley, P. B., \& Calhoun, K. S. (1987). Relaxation training, in vivo exposure and response-prevention in the treatment of compulsive video-game playing.
Cognitive Behaviour Therapy, 16(4), 185190.

https://doi.org/10.1080/16506078709455801

42. Kuss, D. J., \& Griffiths, M. D. (2012a). Online gaming addiction in children and adolescents: A review of empirical research. Journal of behavioral addictions, 1(1), 3-22. https://doi.org/10.1556/jba.1.2012.1.1

43. Kuss, D. J., \& Griffiths, M. D. (2012b). Internet and gaming addiction: a systematic literature review of neuroimaging studies. Brain sciences, 2(3), 347-374. https://doi.org/10.3390/brainsci2030347

44. Lüthi, A., \& Lüscher, C. (2014). Pathological circuit function underlying addiction and anxiety disorders. Nature neuroscience, $17(12)$, 1635-1643. https://doi.org/10.1038/nn.3849

45. Männikkö, N., Billieux, J., \& Kääriäinen, M. (2015). Problematic digital gaming behavior and its relation to the psychological, social and physical health of Finnish adolescents and young adults. Journal of behavioral addictions, $\quad 4(4), \quad 281-288$. https://doi.org/10.1556/2006.4.2015.040

46. Mentzoni, R. A., Brunborg, G. S., Molde, H., Myrseth, H., Skouverøe, K. J. M., Hetland, J., \& Pallesen, S. (2011). Problematic video game use: estimated prevalence and associations with mental and physical health. Cyberpsychology, behavior, and social networking, 14(10), 591-596. https://doi.org/10.1089/cyber.2010.0260

47. Merikangas, K. R., Mehta, R. L., Molnar, B. E., Walters, E. E., Swendsen, J. D., AguilarGaziola, S., ... \& Kolody, B. (1998). Comorbidity of substance use disorders with mood and anxiety disorders: results of the International Consortium in Psychiatric Epidemiology. Addictive behaviors, 23(6), 893-907. https://doi.org/10.1016/s03064603(98)00076-8

48. Molina, J., Sandín, B., \& Chorot, P. (2014). Sensibilidad a la ansiedad y presión psicológica: Efectos sobre el rendimiento deportivo en adolescentes. Cuadernos de Psicología del Deporte, 14(1), 45-54. https://doi.org/10.4321/s157884232014000100006 


\section{López-Mora et al.}

49. Mutz, J., Clough, P.J., \& Pagageorgiou, K.A. (2017). Do individual differences in emotion regulation mediate the relationship between mental toughness and symptoms of depression? Journal of Individual Differences, $\quad 38, \quad 71-82$. https://doi.org/10.1027/1614-0001/a000224

50. OMS (2018). Clasificación International de Enfermedades. Undécima Edición Ginebra.

51. Orús, A. (29 de abril de 2020) Industria del videojuego en España - Datos estadísticos. Statista.

https://es.statista.com/temas/2851/industriadel-videojuego-en-espana/

52. Philip B. \& Kommers, P. A. M. (2005). Gender differences and styles in the use of digital games. Educational Psychology, 25(1), 13-41.

https://doi.org/10.1080/01443410420002948 77

53. Prot, S., \& Gentile, D. A. (2014). Applying risk and resilience models to predicting the effects of media violence on development. Advances in child development and behavior, 46, 215-244. https://doi.org/10.1016/b978-012-800285-8.00008-x

54. Rideout, V., \& Robb, M. B. (2019). The Common Sense census: Media use by tweens and teens. Common Sense Media.

55. Sanderson, J., Browning, B., \& DeHay, H. (2020). " It's the universal language:" Investigating student-athletes' use of and motivations for playing Fortnite. Journal of Issues in Intercollegiate Athletics, 13, 22-44.

56. Sandín, B., Valiente, R. M., Chorot, P., \& Germán, M. A. S. (2007). ASI-3: Nueva escala para la evaluación de la sensibilidad a la ansiedad. Revista de Psicopatología y Psicología Clínica, 12(2), 91-104. https://doi.org/10.5944/rppc.vol.12.num.2.20 07.4036

57. Schaefer, J., Vella, S. A., Allen, M. S., \& Magee, C. A. (2016). Competition anxiety, motivation, and mental toughness in golf. Journal of applied sport psychology, 28(3), 309-320.

https://doi.org/10.1080/10413200.2016.1162 219
58. Sepkowitz, L. (2018, March 20). 'It took over my life': Inside NBA players Fortnite addiction. Retrieved from https://bleacherreport.com/articles/2765469it-took-my-life-over-insidenba-playersfortnite-addiction

59. Soleimani, M. A., Pahlevan Sharif, S., Yaghoobzadeh, A., \& Ong, S. (2016). Relationship between hardiness and addiction potential in medical students. Iranian journal of psychiatry and behavioral sciences, 10(4), e6225. https://doi.org/10.17795/ijpbs-6225

60. Stewart, S. H., \& Kushner, M. G. (2001). Introduction to the special issue on "Anxiety Sensitivity and Addictive Behaviors". Addictive Behaviors, 26(6), 775-785. https://doi.org/10.1016/s03064603(01)00236-2

61. Stockdale, L., \& Coyne, S. M. (2018). Video game addiction in emerging adulthood: Crosssectional evidence of pathology in video game addicts as compared to matched healthy controls. Journal of affective disorders, 225, 265-272.

https://doi.org/10.1016/j.jad.2017.08.045

62. Taylor, S., Zvolensky, M. J., Cox, B. J., Deacon, B., Heimberg, R. G., Ledley, D. R., y Cárdenas, S. J. (2007). Robust dimensions of anxiety sensitivity: development and initial validation of the Anxiety Sensitivity Index-3. Psychological Assessment, 19, 176-188. https://doi.org/10.1037/1040-3590.19.2.176

63. Thelwell, R., Weston, N., \& Greenlees, I. (2005). Defining and understanding mental toughness within soccer. Journal of applied sport psychology, 17(4), 326-332. https://doi.org/10.1080/10413200500313636

64. Thomas, A. C., Sullivan, G. B., \& Allen, F. C. L. (2009). A theoretical model of EGM problem gambling: More than a cognitive escape. International Journal of Mental Health and Addiction, 7(1), 97-107. https://doi.org/10.1007/s11469-008-9152-6

65. Van Rooij AJ, Schoenmakers TM, Vermulst AA, Van den Eijnden RJ, Van de Mheen D. (2011). Online video game addiction: identification of addicted adolescent gamers. Addiction, 106(1), 205-212. https://doi.org/10.1111/j.13600443.2010.03104.x 


\section{Sensibilidad a la ansiedad dureza mental y adicción a videojuegos en deportistas}

66. Vella, K., Johnson, D., Cheng, V. W. S., Davenport, T., Mitchell, J., Klarkowski, M., \& Phillips, C. (2019). A sense of belonging: Pokémon GO and social connectedness. Games and Culture, 14(6), 583-603. https://doi.org/10.1177/1555412017719973

67. Vondráčková, P., \& Gabrhelík, R. (2016). Prevention of Internet addiction: A systematic review. Journal of Behavioral Addictions, 5(4), $568-579$. https://doi.org/10.1556/2006.5.2016.085

68. Wang, J. J., Wang, X., \& Li, L. Y. (2014). The relationship between College Students' Internet addiction and mental toughness. Theory Research, 03.

69. Ward, M. R. (2018). Cutting class to play video games. Information Economics and Policy, 42, 11-19. https://doi.org/10.1016/j.infoecopol.2017.10. 001

70. Wartberg, L., Kriston, L., \& Thomasius, R. (2020). Internet gaming disorder and problematic social media use in a representative sample of German adolescents: Prevalence estimates, comorbid depressive symptoms and related psychosocial aspects. Computers in Human Behavior, 103, 31-36. https://doi.org/10.1016/j.chb.2019.09.014

71. Weiser, E. B. (2000). Gender differences in Internet use patterns and Internet application preferences: A two-sample comparison. Cyberpsychology and behavior, 3(2), 167178. https://doi.org/10.1089/109493100316012

72. Woodman, T., \& Hardy, L. (2001). Stress and anxiety. In R. Singer, H. A. Hausenblas, \& C. M. Janelle (Eds.), Handbook of research on sport psychology (pp. 290-318). Wiley.

73. Zalewska, A. M., Krzywosz-Rynkiewicz, B., Clough, P. J., \& Dagnall, N. (2019). Mental toughness development through adolescence: Effects of age group and community size. Social Behavior \& Personality: An International Journal, 47(1), 1-8. https://doi.org/10.2224/sbp.7376 